\title{
CAPS2 Deficiency Impairs the Release of the Social Peptide Oxytocin, as Well as Oxytocin-Associated Social Behavior
}

\author{
Shuhei Fujima, ${ }^{1}$ Ryosuke Yamaga, ${ }^{1}$ Haruka Minami, ${ }^{1}$ Shota Mizuno, ${ }^{1}$ Yo Shinoda, ${ }^{2}{ }^{\text {DTetsushi Sadakata, }}{ }^{3}$ \\ ${ }^{\circledR}$ Manabu Abe, ${ }^{4}$ Kenji Sakimura, ${ }^{4}$ Yoshitake Sano, ${ }^{1}$ and ${ }^{\circledR}$ Teiichi Furuichi ${ }^{1}$ \\ ${ }^{1}$ Department of Applied Biological Science, Faculty of Science and Technology, Tokyo University of Science, Chiba 278-8510, Japan, ${ }^{2}$ Department of \\ Environmental Health, School of Pharmacy, Tokyo University of Pharmacy and Life Sciences, Tokyo 192-0392, Japan, ${ }^{3}$ Education and Research \\ Support Center, Gunma University Graduate School of Medicine, Maebashi, Gunma 371-8511, Japan, and ${ }^{4}$ Department of Cellular Neurobiology, \\ Brain Research Institute, Niigata University, Niigata 951-8585, Japan
}

$\mathrm{Ca}^{2+}$-dependent activator protein for secretion 2 (CAPS2) regulates dense-core vesicle (DCV) exocytosis to facilitate peptidergic and catecholaminergic transmitter release. CAPS2 deficiency in mice has mild neuronal effects but markedly impairs social behavior. Rare de novo Caps2 alterations also occur in autism spectrum disorder, although whether CAPS2-mediated release influences social behavior remains unclear. Here, we demonstrate that CAPS2 is associated with DCV exocytosis-mediated release of the social interaction modulatory peptide oxytocin (OXT). CAPS2 is expressed in hypothalamic OXT neurons and localizes to OXT nerve projection and OXT release sites, such as the pituitary. Caps2 KO mice exhibited reduced plasma albeit increased hypothalamic and pituitary OXT levels, indicating insufficient release. OXT neuron-specific Caps 2 conditional KO supported CAPS2 function in pituitary OXT release, also affording impaired social interaction and recognition behavior that could be ameliorated by exogenous OXT administered intranasally. Thus, CAPS2 appears critical for OXT release, thereby being associated with social behavior.

Key words: CAPS2/Cadps2; oxytocin; social behavior; dense-core vesicle; paraventricular nucleus; pituitary

Significance Statement

The role of the neuropeptide oxytocin in enhancing social interaction and social bonding behavior has attracted considerable public and neuroscientific attention. A central issue in oxytocin biology concerns how oxytocin release is regulated. Our study provides an important insight into the understanding of oxytocin-dependent social behavior from the perspective of the CAPS2-regulated release mechanism.

Received Dec. 30, 2020; revised Mar. 4, 2021; accepted Apr. 2, 2021.

Author contributions: S.F., R.Y., H.M., Y.S, T.S., M.A., K.S., Y.S., and T.F. designed research; S.F., R.Y., H.M., S.M., and M.A. performed research; S.F., R.Y., S.M., Y.S., M.A., and K.S. contributed unpublished reagents/ analytic tools; S.F., R.Y., H.M., S.M., Y.S., and T.F. analyzed data; and S.F., T.S., Y.S., and T.F. wrote the paper.

This work was supported by Grants-in-Aid for KAKENHI (17H03563, 17K19638, 26290026, 15K14356, 15K21013, 15K08192) from the Japan Society for the Promotion of Science and the Ministry of Education, Culture, Sports, Science, and Technology of Japan, and a research grant from the NOVARTIS Foundation (Japan) for the Promotion of Science. Generation of the recombinant mouse lines was supported by a Grantin-Aid for Scientific Research on Innovative Areas (Comprehensive Brain Science Network) from the Ministry of Education, Science, Sports and Culture of Japan. We thank Rina Maniwa, Natsuki Amemiya, Kazuki Shimizu, and Jun-na Hirota for technical support.

S. Fujima's present address: Kobe University, Graduate School of Medicine, Kobe 657-8501, Japan.

M. Abe's present address: Department of Animal Model Development, Brain Research Institute, Niigata University, Niigata 951-8585, Japan.

K. Sakimura's present address: Department of Animal Model Development, Brain Research Institute, Niigata University, Niigata 951-8585, Japan.

The authors declare no competing financial interests.

Correspondence should be addressed to Teiichi Furuichi at tfuruichi@rs.tus.ac.jp.

https://doi.org/10.1523/JNEUROSCI.3240-20.2021

Copyright $\odot 2021$ the authors

\section{Introduction}

The $\mathrm{Ca}^{2+}$-dependent activator protein for secretion (CAPS) protein family consists of two members, CAPS1 and CAPS2, and regulates the exocytosis of dense-core vesicles (DCVs) that contain peptidergic and monoaminergic transmitters (Grishanin et al., 2002; Sadakata et al., 2007a; Liu et al., 2008). We previously identified CAPS2 as a regulator for the release of brain-derived neurotrophic factor (BDNF) in the mouse brain. Caps2-knockout (KO) mice showed decreased release of BDNF and consequent impairments in neuronal development and synaptic function (Sadakata et al., 2004; Shinoda et al., 2011). Notably, the KO mice also exhibited impaired behavioral phenotypes, including reduced sociality, increased anxiety levels, and defective circadian rhythm (Sadakata et al., 2007a). Moreover, through genomic analysis of patients with autism spectrum disorder (ASD), we found that some Japanese ASD patients exhibited aberrantly increased expression of an alternative splicing Caps2 isoform (Caps2-dex3) detrimental to axonal transport (Sadakata et al., 2007a), and mice expressing Caps2-dex3 had impaired social behavior (Sadakata et al., 2012). A possible link between 
A

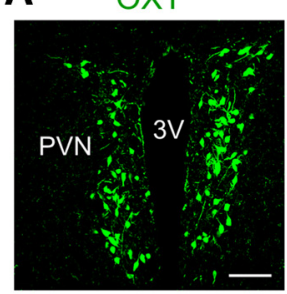

B

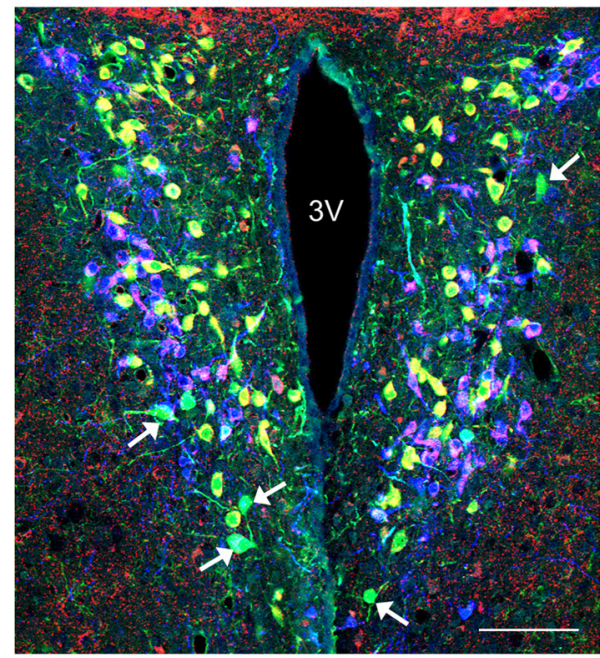

CAPS2

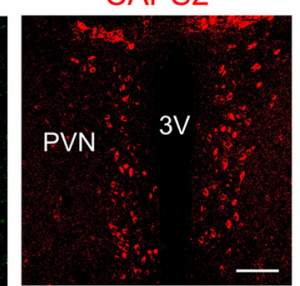

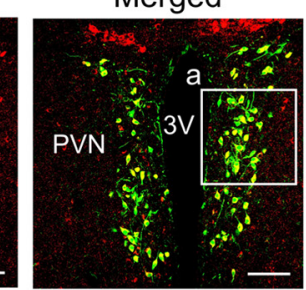

C

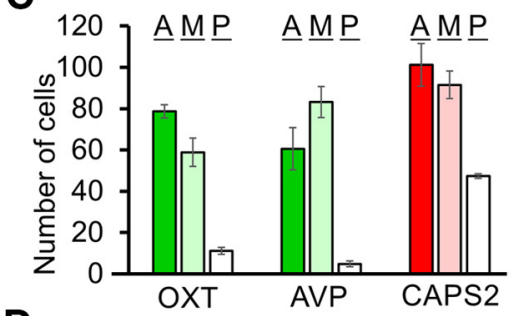

D

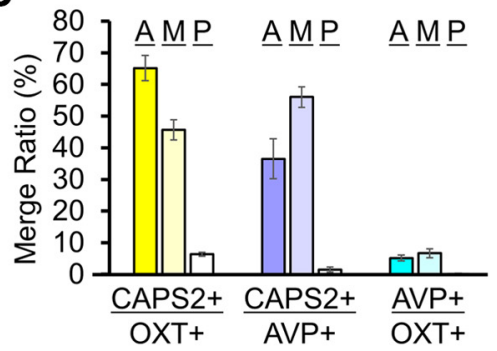

Figure 1. Expression of CAPS2 in OXT and AVP neurons of the hypothalamic PVN. $\boldsymbol{A}$, Representative images of coronal PVN sections coimmunostained for OXT (green) and CAPS2 (red, upper row) and CAPS2 (red, bottom row). The merged images are shown. Magnified views of the boxed areas a of merged images are shown on the right. Scale bar, $100 \mu \mathrm{m} ;$ magnified images, $50 \mu \mathrm{m}$. 3V, Third ventricle. B, Triple-merged image for OXT (green), AVP (blue), and CAPS2 (red) immunoreactivity at the position -0.75 mm to Bregma. Arrows indicate 0XT-immunopositive cells with very low or undetectable levels of CAPS2 immunoreactivity. C, Number of OXT-, AVP-, and CAPS2-single-positive cells in the anterior, middle, and posterior areas of the PVN. Anterior: OXT: $78.67 \pm 3.18$; AVP: $60.5 \pm$ 10.25; CAPS2: $101.17 \pm$ 10.31. Middle: OXT: $58.83 \pm 6.87$; AVP: $83.17 \pm 7.42$; CAPS2: $91.5 \pm 6.6$. Posterior: OXT: $11.17 \pm 1.69 ;$ AVP: $4.83 \pm 1.36$; CAPS2: $47.33 \pm 1.17$. For both WT and Caps2-KO mice $N=3 ; 1-2$ coronal sections were used for analysis of each part. $D$, Ratio of OXT-, AVP-, and CAPS2-double-positive cells in the anterior, middle, and posterior areas of the PVN. Ratio of OXT/CAPS2 double positive per OXT single positive, of AVP/CAPS2 double positive per AVP single positive, and of OXT/AVP double positive per OXT single positive are $65.17 \pm 3.94 \%, 36.50 \pm 6.33 \%$, and $5.17 \pm 0.93 \%$ (anterior); $45.67 \pm 3.17 \%, 56 \pm 3.21 \%$, and $6.67 \pm 1.36 \%$ (middle); and $6.34 \pm 0.6 \%, 1.5 \pm 0.76 \%$, and $0.17 \pm 0.17 \%$ (posterior), respectively. One to 2 coronal sections were used for analysis of each part (WT $N=3$ and $K O N=3$ ). Data represent the means $\pm S E M$. Anterior (A): Bregma -0.70 $\mathrm{mm}$, medial (M): Bregma $-0.90 \mathrm{~mm}$, and posterior (P): Bregma $-1.1 \mathrm{~mm}$.

ASD and alteration of the Caps2 gene has also been suggested by multiple studies reporting the identification of de novo and rare copy number variations and single nucleotide variations in the Caps2 gene of some ASD patients (Sadakata et al., 2007b; Christian et al., 2008; Marshall et al., 2008; Stein et al., 2010; Okamoto et al., 2011; Girirajan et al., 2013; Bonora et al., 2014; Aristidou et al., 2017; Grabowski et al., 2017; Grove et al., 2019). However, it remains unclear whether the CAPS2-mediated release mechanism is associated with physiological processes for sociality expression. It is also unclear which secretory substance (s) are influenced by CAPS2 deficiency, which may contribute to the risk of ASD.

Oxytocin (OXT) is a neuropeptide that is produced in the hypothalamus. OXT neurons project their axons to the neurohypophysis, that is, the posterior pituitary lobes, to release OXT peripherally into the blood circulation as the peptide hormone; these neurons also innervate several brain regions where they release OXT as a neuropeptide (Ross and Young, 2009; Stoop, 2012; Baribeau and Anagnostou, 2015; Johnson and Young, 2017). Thus, OXT is involved in regulating not only peripheral functions, including uterine contraction and lactation, but also central nervous functions, including social, aggressive, fear, and maternal behaviors (Knobloch et al., 2012; Marlin et al., 2015; Hung et al., 2017; Lin et al., 2018; Hasan et al., 2019). Recently, the effect of exogenously applied OXT on social behavior has attracted considerable attention as a social peptide: in particular, the administration of OXT not only restores social deficits in an ASD mouse model (Peñagarikano et al., 2015; Sgritta et al., 2019) but also increases emotional recognition in humans and improves social and repetitive behavior in ASD (Kosfeld et al., 2005; Auyeung et al., 2015). Consistent with these observations, it has also been shown that the blood OXT concentration is slightly lower in ASD patients than in healthy subjects (Zhang et al., 2016) and that Oxt and Oxt receptor KO mice show abnormalities in social behaviors (Ferguson et al., 2000; Takayanagi et al., 2005; Crawley et al., 2007; Lazzari et al., 2013). However, the molecular mechanism underlying OXT release remains to be elucidated.

We previously reported that the CAPS2 protein is expressed in the hypothalamus and pituitary gland (Sadakata et al., 2006). We therefore hypothesized that the deficiency of CAPS2 in mice affects OXT release, thereby resulting in impaired social behavior. In this study, we aimed to reveal whether CAPS2 is expressed in OXT-producing neurons of the hypothalamic paraventricular nucleus (PVN) and posterior pituitary. Moreover, by using both Caps2 $\mathrm{KO}$ and OXT neuron-specific Caps2 conditional $\mathrm{KO}$ (cKO) mice, we addressed whether the CAPS2 deficiency causes impairments in OXT release and social behavior. We also 
A

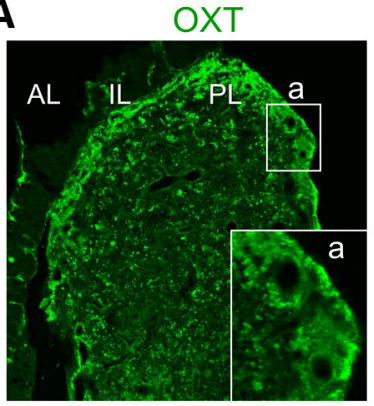

CAPS2

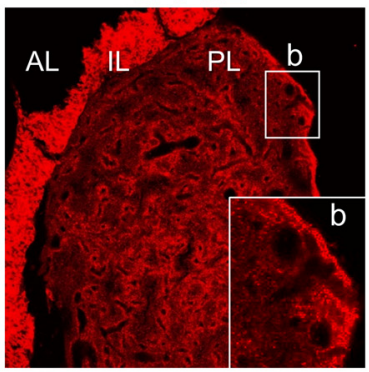

Merge

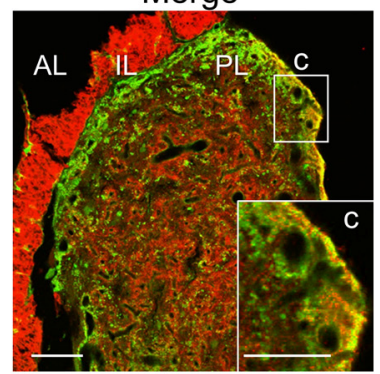

B

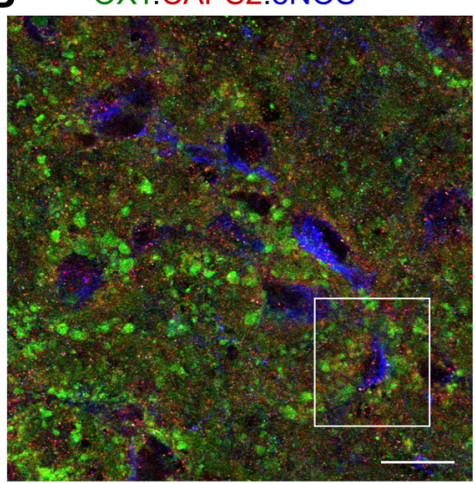

C
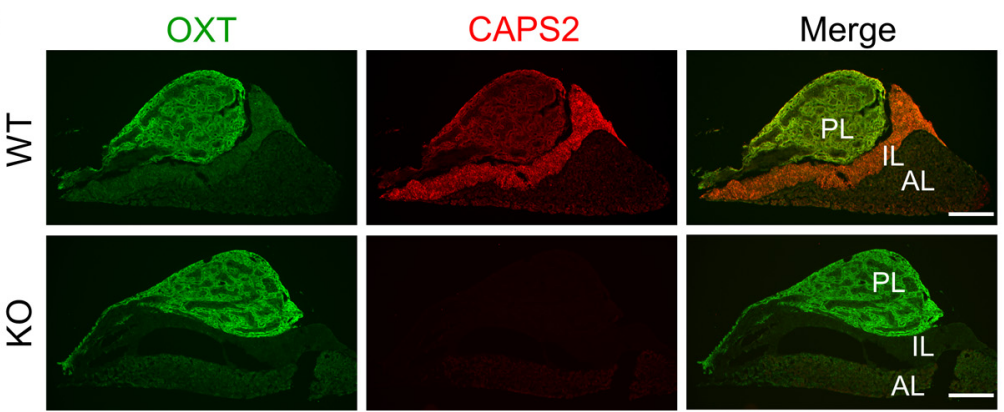

Figure 2. Distribution of CAPS2 in OXT-projecting sites of the posterior pituitary. $A$, Representative images of coronal pituitary sections immunostained for 0XT (green) and CAPS2 (red). The merged image is shown on the right. Scale bar, $100 \mu \mathrm{m}$. Magnified views of boxed areas a, b, and c are shown as insets a-c on the right bottom corners. Scale bar, $50 \mu \mathrm{m}$. $\boldsymbol{B}$, Representative image of a posterior pituitary section coimmunostained for OXT (green), CAPS2 (red), and eNOS (blue) is shown on the left. Scale bar, $20 \mu \mathrm{m}$. Three magnified views for 0XT/eNOS-, CAPS2/ eNOS- and OXT/CAPS2/eNOS-immunostaining of the boxed area are shown on the right. CAPS2 immunosignals are located around the areas near the e-NOS-positive blood vessel, and some puncta immunopositive for OXT and CAPS2 are observed as adjacent to each other. Scale bar, $50 \mu \mathrm{m}$. bv, Blood vessel. C, Representative images of pituitary sections immunostained for OXT (green) and CAPS2 (red) in WT (upper row) and Caps2 KO (lower row). The merged images are shown on the right. Scale bar, $200 \mu \mathrm{m}$. AL, Anterior lobe; IL, intermediate lobe; PL, posterior lobe.

evaluated whether exogenously applied OXT could ameliorate the impaired social behavior consequent to a loss of CAPS2 function in mice. Collectively, our study provides the first evidence highlighting the significance of CAPS2 in the OXT release mechanism crucial for social behavior.

\section{Materials and Methods}

Animals. We used male Caps2 KO mice (Sadakata et al., 2007a) and wild-type (WT) littermate controls in this study. To generate OXT neuron-specific Caps $2 \mathrm{cKO}$ mice by using the Oxt gene promoter-driven Cre/loxP site-specific recombination system, we created two mouse lines, Oxt-iCre knock-in (KI) mice (C57BL/6-Oxt ${ }^{\text {tm1.1(Cre)Ksak }}$, hereafter written as $\left.\mathrm{Oxt}^{\text {cre/+}}\right)$, which carry an insert sequence of the self-processing furin-2A in front of the improved Cre (iCre) recombinase at the end of the coding sequence of the Oxt gene, and Caps2-flox mice (C57BL/6Cadps $2^{\text {tmI.1Ksak }}$, hereafter written as Caps $2^{\text {flox/+ }}$ ), which carry the loxPflanked exon6 of the Caps2 gene. We mated Caps $2^{\text {flox/flox }}$ female mice with Caps $2^{\text {flox/+ }} \mathrm{Oxt} \mathrm{cre}^{\text {cre }}$ male mice to produce Caps $2^{\text {flox/flox }} \mathrm{Oxt}^{+/+}$as a control and Caps $2^{\text {flox/flox }} \mathrm{Oxt} \mathrm{cre}^{\text {cre }}$ for Caps2 cKO mice. All mice used (3-6 months old) were group housed and maintained under a 12:12 light-dark cycle. The experiments were approved by the Institutional Animal Care and Use Committee of Tokyo University of Science (approval reference no. N18003/4, N19004/5, N20004/5). All experiments were conducted under the Regulations for Animal Research of the Tokyo University of Science.

Immunohistochemistry. Mice were deeply anesthetized using somnopentyl (Kyoritsu Seiyaku) perfused transcardially with saline followed by $4 \%$ paraformaldehyde (PFA) in phosphate-buffered saline (PBS). Brains and pituitaries were dissected, postfixed in 4\% PFA overnight, and subsequently immersed in 30\% sucrose in PBS for cryoprotection. Following the fixation and cryoprotection, tissues were embedded in O.C.T compound (catalog \#4583, Sakura Finetek) and frozen via a frozen tissue block preparation apparatus (Histo-Tek PINO-600, Sakura Finetek). The brains and pituitary were sectioned coronally at a thickness of $30 \mu \mathrm{m}$. Sections of brains were stored at $-20^{\circ} \mathrm{C}$ in cryoprotection solution (30\% ethylene glycol, $25 \%$ glycerol in $1 \times$ PBS), and sections of the pituitary were attached to glass slides (catalog \#S9445, Matsunami Glass) and stored at $-80^{\circ} \mathrm{C}$.

In IHC, stored brains and pituitaries were washed using PBST $(0.2 \%$ Triton in PBS) and subsequently blocked with blocking solution [5\% normal donkey serum (catalog \#S30-100ML, Chemicon) in PBST] for 1 
A

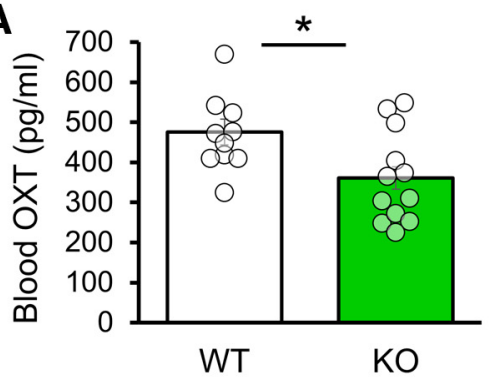

C
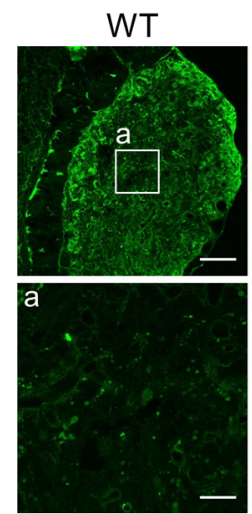

E

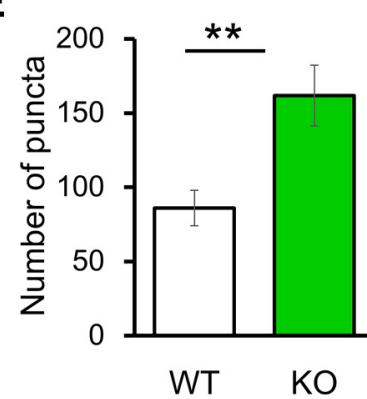

Caps2 KO

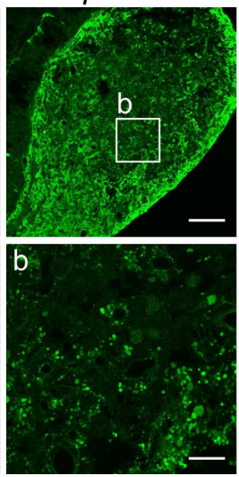

$\mathbf{F}$

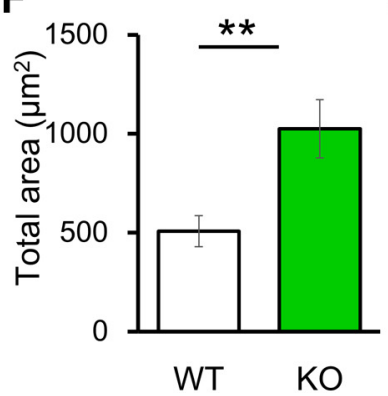

D
B
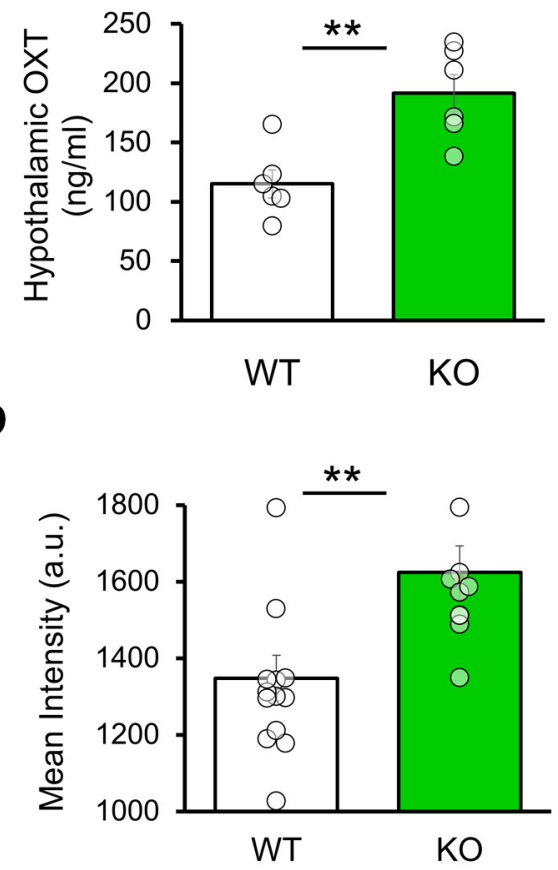

G

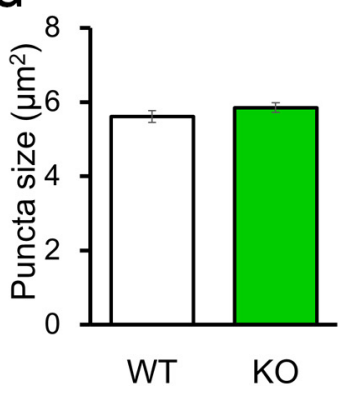

Figure 3. Changes of OXT levels in the blood and pituitary of Caps2 KO mice. A, Plasma OXT concentrations as calculated using ELISA. WT ( $N=10 \mathrm{mice}): 475.99 \pm 32.42 \mathrm{pg} / \mathrm{ml})$, Caps2 KO ( $N=13$ mice): $360.92 \pm 28.08 \mathrm{pg} / \mathrm{ml}, p=0.025$, Student's $t$ test. $\boldsymbol{B}$, Hypothalamic PVN OXT concentrations as calculated using ELISA. WT ( $N=6 \mathrm{mice}): 115.0 \pm 11.70 \mathrm{ng} / \mathrm{ml}$, Caps2 KO (N=6 mice): $191.41 \pm 15.66 \mathrm{ng} / \mathrm{ml}, p=0.0029$, Student's $t$ test. C, Representative images of posterior pituitary sections immunostained for OXT (green) in WT (left) and (aps2 KO (right) mice. Scale bars: $100 \mu \mathrm{m}(20 \times), 20 \mu \mathrm{m}(100 \times)$. D, Mean intensity of immunofluorescence for posterior pituitary OXT. WT ( $N=13$ mice): $1347.99 \pm 70.04$ a.u., Caps2 K0 ( $N=10$ mice): $1623.60 \pm 59.44$ a.u., $p=0.0095$, Student's $t$ test. A total of $6-9$ pituitary sections were analyzed for each mouse tested. $\boldsymbol{E}$, Number of OXT puncta of immunofluorescence for the posterior pituitary. WT ( $N=13$ mice): $85.96 \pm 12.01$, Caps $2 \mathrm{KO}(N=10$ mice): $161.79 \pm 20.54$. $p=0.0034$, Student's $t$ test. A total of $6-9$ pituitary sections were analyzed for each mouse tested. $\boldsymbol{F}$, 0XT puncta area of immunofluorescence for the posterior pituitary. WT ( $N=13$ mice): $508.11 \pm 78.15 \mu \mathrm{m}^{2}$, Caps2 $\mathrm{KO}$ ( $N=10$ mice): $1025.24 \pm 146.09 \mu \mathrm{m}^{2}, p=0.0038$, Student's $t$ test. A total of 6-9 pituitary sections were analyzed for each mouse tested. $G$, OXT puncta size of immunofluorescence for the posterior pituitary. WT ( $N=13$ mice): $5.61 \pm 0.16$, Caps2 $\mathrm{KO}$ ( $N=10$ mice): $5.85 \pm 0.13, p=0.28$, Student's $t$ test. A total of 6-9 pituitary sections were analyzed for each mouse tested. $* p<0.05, * * p<0.01$.

h. Subsequently, sections were reacted with primary antibody diluted with blocking solution overnight. Sections were washed with PBST three times and subsequently reacted with secondary antibody diluted with blocking solution. Then, sections were reacted with DAPI $(1 \mathrm{ng} / \mathrm{ml}$; cata$\log$ \#D1306, Thermo Fisher Scientific) in PBST for $30 \mathrm{~min}$ and washed twice with PBST. Finally, sections were mounted with Prolong Diamond (Invitrogen). Digital images were obtained by confocal microscopy (FluoView FV1000, Olympus).

For detecting CAPS family proteins, guinea pig anti-CAPS2 antibody (1:100) and rabbit anti-CAPS1 antibody (1:1000) were used (Sadakata et al., 2004, 2007b). As the other primary antibodies, we used mouse antiOXT antibody (1:2000; catalog \#MAB5296, Abcam), rabbit antiarginine vasopressin (AVP) antibody (1:2000; catalog \#AB1565, Millipore) rat anti-mCherry antibody (1:5000; catalog \#M11217, Invitrogen), and mouse anti-eNOS antibody (1:500; catalog \#610296, BD Biosciences). As secondary antibodies, we used Alexa Fluor 488 Donkey anti-Mouse IgG (H+L; 1:1000; catalog \#A21202, Invitrogen), Alexa Fluor 488 Goat anti-Guinea Pig IgG $(\mathrm{H}+\mathrm{L})$ highly cross-adsorbed (1:500; catalog \#A11073, Invitrogen), Alexa
Fluor 555 Donkey anti-Goat IgG (H+L; 1:1000; catalog \#A21432, Invitrogen), Alexa Fluor 488 Chicken anti-Rabbit IgG $(\mathrm{H}+\mathrm{L}$; 1:300; catalog \#A21441, Invitrogen), and Alexa Fluor 647 Goat Anti-Rabbit IgG (H+L; 1:300; catalog \#A21244, Invitrogen).

OXT ELISA. We used 8-week-old Caps2 KO and WT littermates for measurements of blood OXT levels. Mice were anesthetized using isoflurane, and then we collected blood samples from the right ventricle of mice using a syringe treated with heparin sodium (catalog \#3334401A6077, Ajinomoto). Subsequently, blood samples were centrifuged at $1000 \times g$ for $15 \mathrm{~min}$, and the supernatant was collected as blood plasma. We diluted plasma samples eightfold with assay buffer and measured OXT levels using the Oxytocin ELISA kit (catalog \#ADI-900153A, Enzo Life Sciences).

Open-field test. Locomotor activity was measured in an open field $(46 \times 30 \mathrm{~cm})$. Each mouse (3-month-old males) was placed in the center of the open field, and its movements were monitored for 10 min using a camera (C270N HD Webcam, Logicool). The total distance traveled, average speed, time spent at the center, and time spent at the corners were analyzed using video tracking software (ANY-Maze, Stoelting). 
Reciprocal social interaction test. The sociability of Caps $2 \mathrm{cKO}$ and control Caps $2^{\text {flox/flox }}$ male mice (3-5 months old) were tested. The experimental scheme for reciprocal social interaction was primarily based on a previous report regarding Oxt $\mathrm{KO}$ mice (Ferguson et al., 2000) with modification of single housing time and interaction time for our mice to obtain stable and reproducible results. All experimental mice were single housed for $2 \mathrm{~d}$ before the social interaction test. A test male mouse was placed in a cage to habituate itself for $20 \mathrm{~min}$. Then, an unfamiliar male mouse (C57BL/6J, the same genetic background of the two test mice, 3 months old) was introduced into the cage of the test mouse. Social interaction trials were conducted for 3 min each and repeated three times with an intertrial interval (ITI) of $10 \mathrm{~min}$, followed by an additional 3 min interaction with the second unfamiliar C57BL/6J mouse at $10 \mathrm{~min}$ after the third trial. Social behavior was monitored using a camera (C270N HD Webcam, Logicool) and analyzed for reciprocal interaction (including sniffing, approaching, and following).

Stereotaxic surgery. For surgery, Oxt-iCre mice and C57BL/6J mice were used. All mice were anesthetized by intraperitoneal injection of pentobarbital $(80 \mathrm{mg} / \mathrm{kg}$ of body weight; Kyoritsu Seiyaku) and subcutaneously injected with dexamethasone $(0.2 \mathrm{mg} / \mathrm{kg}$ of body weight; 11107-51; Nacalai Tesque) and Rimadyl (carprofen; $5 \mathrm{mg} / \mathrm{kg}$ of body weight; Zoetis) in PBS as a pain killer, then mice were placed in a stereotaxic apparatus (Angle Two, Computer-Assisted Stereotaxic System, Leica Microsystems). For anterograde tracing of OXT neurons, recombinant adeno-associated virus (AAV5-DIO-ChR2-mCherry; $5.2 \times 1012$ virus molecules/ml; UNC Vector Core) was infused into the PVN (anterior-posterior: $-0.75 \mathrm{~mm}$, mediolateral: $\pm 0.3 \mathrm{~mm}$, dorsoventral: $-5.0 \mathrm{~mm}$ ).

OXT intranasal infusion. Previous reports indicated that intranasal administration of OXT could effectively reach the brain and improve social behavior in ASD model mice (Neumann et al., 2013; Peñagarikano et al., 2015). In the present study, we conducted experiments regarding OXT administration before performing the social interaction test. We used $2.4 \times \mathrm{g} / \mathrm{l}$ OXT (catalog \#4084-v, Peptide Institute) diluted in PBS. We intranasally administered $2.5 \mu \mathrm{l}$ OXT solution $(6.0 \mu \mathrm{g} \mathrm{OXT})$ or PBS to Caps $2 \mathrm{cKO}$ and control mice. It has been reported that intranasal OXT administration reaches its peak level in the brain in $15 \mathrm{~min}$ and markedly decreases after 75 min (Neumann et al., 2013). All mice were therefore treated with OXT or PBS 20 min before the social interaction test. This experimental scheme is in accordance with a previous report involving the rescue of social abnormalities in ASD model mice (Peñagarikano et al., 2015; Sgritta et al., 2019).

Microscopy. A fluorescence microscope (Eclipse Ti, Nikon) equipped with an EM-CCD camera (iXon+, Andor) and confocal laser scanning microscope (FluoView FV1000, Olympus) were used to observe fluorescence images and obtain their digital data, respectively.

Statistical analysis. All digital image data were obtained and analyzed using Fiji ImageJ. Video data of social interaction tests were blinded and manually analyzed. Statistical data were assessed using the two-tailed Student's $t$ test with Excel and two-way ANOVA, followed by the Tukey-Kramer post hoc test using StatView (SAS Institute). Signifiers of $p$ values used are as follows: $p>0.05$, not significant; $p \leq 0.05 ; p \leq 0.01$; $p \leq 0.001$. Unless otherwise indicated, all data are presented as the mean \pm SEM. The number of samples used are shown in the figure legends.

\section{Results}

CAPS2 protein is expressed in OXT and AVP neurons of the hypothalamic PVN

To address the potential of CAPS2-dependent regulation of OXT release, we first analyzed the cellular distribution of CAPS2 in OXT neurons of the hypothalamic PVN by IHC. CAPS2 immunoreactivity was highly localized to OXT-immunopositive cells of the PVN (Fig. 1A). CAPS2 immunoreactivity was also detected in a distinct class of neurons in the PVN that were immunopositive for AVP, a peptide analogous to OXT but differs in 2 of 9 amino acids (Fig. 1B). We further analyzed the distribution of OXT-, AVP-, and CAPS2-immunopositive cells along the anterior-posterior axis of the PVN. Cells immunopositive for OXT, AVP, and CAPS2 were mostly localized to the anterior and middle parts of the tissue specimens analyzed, with the number of OXT-producing cells being slightly larger than that of AVP-producing cells (Fig. 1C). CAPS2 immunoreactivity was more frequently detected in OXT cells of the anterior $(65 \%)$ than the medial part $(45 \%)$, which was reversed for AVP cells (36.5\% anterior versus 56\% medial; Fig. 1D). These results suggest that CAPS2 is involved in DCV release from a majority of OXT cells in the anterior part and AVP cells in the medial part.

\section{CAPS2 is localized in the posterior pituitary at the sites of OXT neuron projection}

The majority of OXT neurons in the PVN project via the hypothalamic-hypophyseal tract to the posterior pituitary lobe and release OXT as a neurohypophyseal peptide hormone from the 


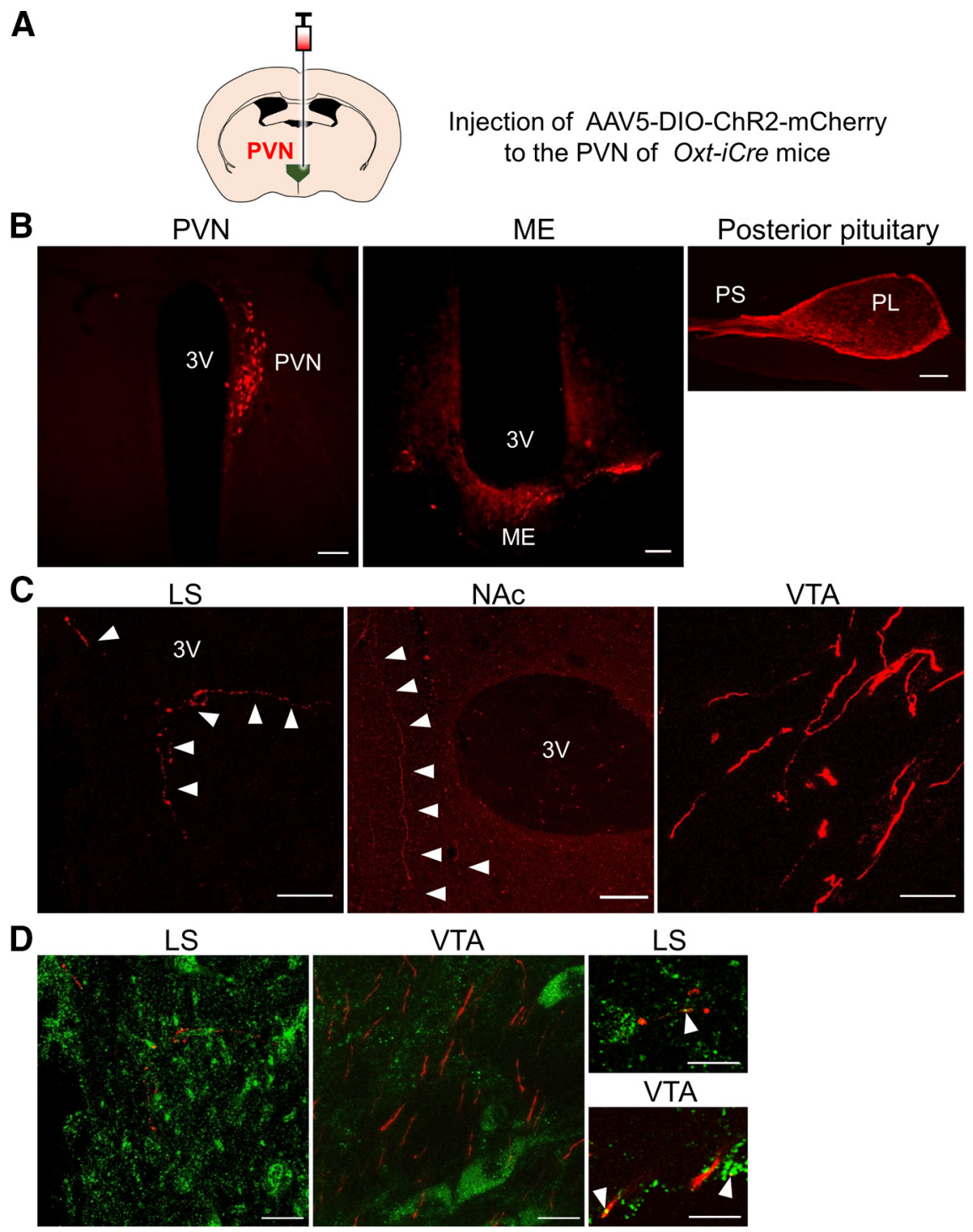

Figure 5. Labeling of OXT projecting neurons by infusing AAV-DIO-mCherry into the PVN of Oxt-iCre mice. A, Model of infusing AAV-DIO-mCherry into the PVN of Oxt-iCre (Oxt ${ }^{\text {(re/+}}$ ) mice. $\boldsymbol{B}$, Representative mCherry images (red) in the PVN, median eminence (ME), and pituitary. C, Representative images for mCherry (red) in brain regions. $\boldsymbol{D}$, Sections of the lateral septum and ventral tegmental area of infected brains were analyzed by mCherry (red) and CAPS2 immunoreactivity (green). Representative magnified views are shown on the right. Scale bar, $100 \mu \mathrm{m}$, except for $13 \mu \mathrm{m}$ in the magnified view of $\boldsymbol{D}$; $3 \mathrm{~V}$, Third ventricle; PS, posterior stalk; PL, posterior lobe of the pituitary; LS, lateral septum; NAc, nucleus accumbens.

axon terminals to the blood circulation. We next investigated the localization of CAPS2 in the pituitary, to which the presynaptic terminals of OXT neurons are innervated. OXT immuno signals were localized to the posterior lobes in the pituitary, whereas CAPS2 immuno signals were distributed at high levels in the intermediate lobe and at medium levels in the posterior lobe (Fig. $2 A$ ), as previously reported (Sadakata et al., 2006). CAPS2-immunoreactive puncta were roughly localized around OXTimmunoreactive puncta (Fig. 2A, magnified views). CAPS2 immunosignals were located around the areas near the blood vessel immunopositive for endothelial nitric oxide synthase (eNOS; Fig. 2B), and a portion of the OXT and CAPS2 immuno signals were also adjacently localized around the areas in the vicinity of eNOS-positive blood vessels (Fig. 2B, magnified views). In contrast, apparent CAPS2 immunoreactivity was not detectable in the pituitary lobe of CAPS2 KO mice (Fig. 2C), supporting our assumption that CAPS2 is produced in either hypothalamic OXT and AVP neurons and transported along OXT or AVP axons to the pituitary. Together, the results suggest the possible involvement of CAPS2 protein in the mechanism underlying OXT secretion into the bloodstream.

\section{CAPS2 deficiency causes a reduction of OXT levels in the blood but not the pituitary}

To verify our assumption that CAPS2 regulates pituitary OXT release into blood vessels, we compared blood OXT levels between Caps2 KO and WT mice using an enzyme-linked immunosorbent assay (ELISA) for OXT. The results showed that the plasma OXT concentration was significantly lower in Caps2 $\mathrm{KO}$ mice than in WT littermates (Fig. 3A; WT: $475.99 \pm 32.42 \mathrm{pg} / \mathrm{ml} ; n=10$; Caps $2 \mathrm{KO}: 360.92 \pm 28.08 \mathrm{pg} / \mathrm{ml}$; $n=13$, Student's $t$ test, $p=0.025)$. In contrast, the OXT concentration of hypothalamic PVN extracts was higher in Caps2 KO mice than WT littermates (Fig. 3B; WT: $115.0 \pm 11.70 \mathrm{ng} / \mathrm{ml}$; $n=6$; Caps2 KO: $191.41 \pm 15.66 \mathrm{ng} / \mathrm{ml} ; n=6$, Student's $t$ test, $p=0.0029)$. In addition, the IHC of pituitary OXT revealed that the immunosignal intensity of the posterior pituitary lobe was much higher in Caps2 KO mice than in WT littermates [Fig. 3C, 
A

Caps2 Targeting cKO

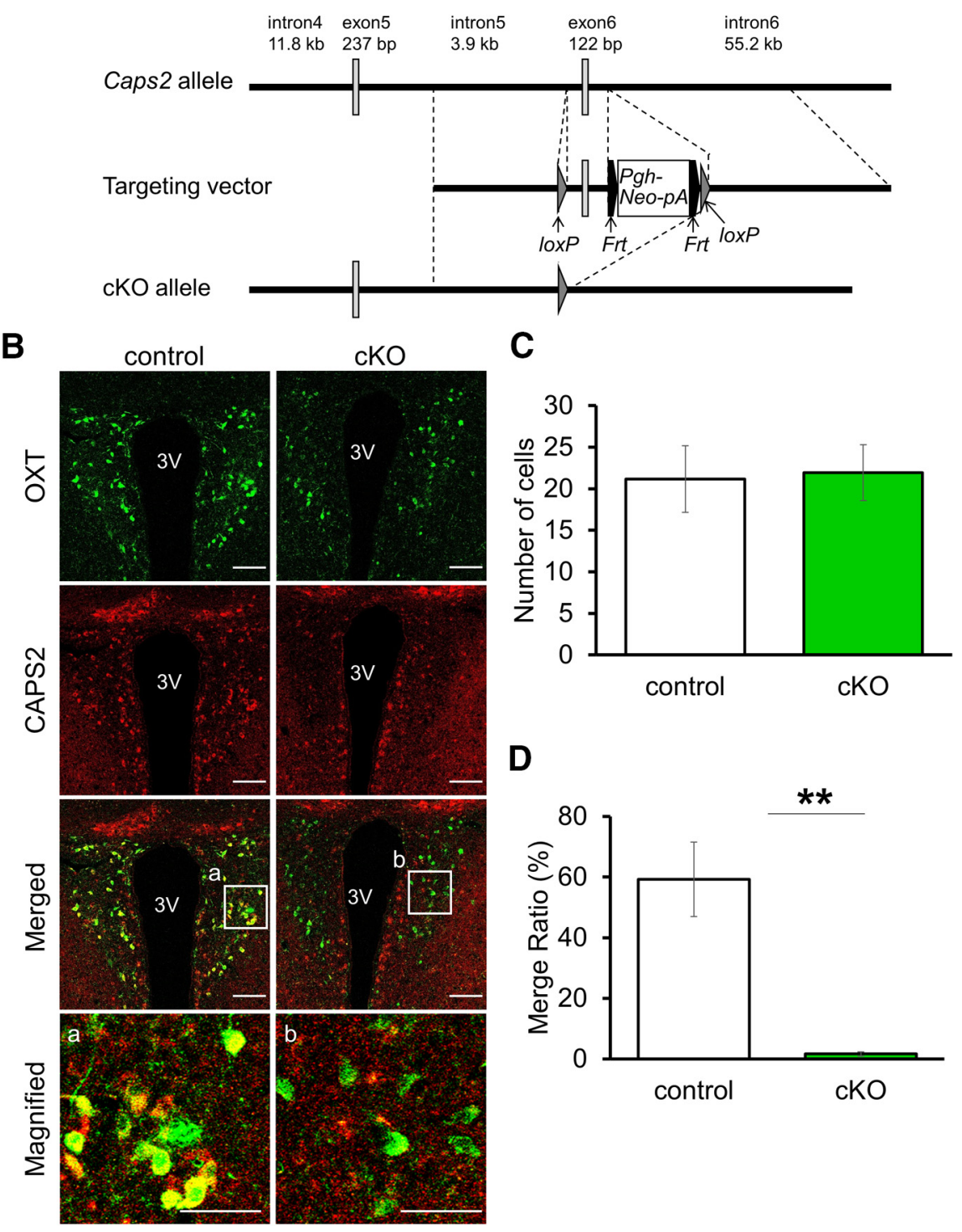

Figure 6. Conditional Caps2 KO in OXT neurons. $A$, Construction of Caps2 CKO mice. The genomic structure of the mouse Caps2 allele (top line), the basic structure of the targeting vector

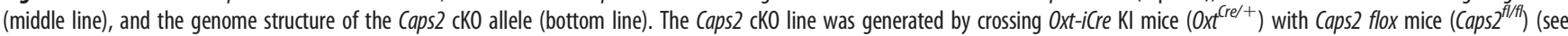
Materials and Methods). B, Representative images of coronal PVN sections immunostained for OXT (green) and CAPS2 (red) in control (Oxt ${ }^{+/+}$, Caps ${ }^{f / f f}$ ) (left) and Caps2 cKO (Oxt $^{\text {(re/+ }}$, Caps $^{f / f f}$ ) (right) mice on the top and second rows, respectively. The merged images and their magnified views inside boxed areas a and $b$ are shown on the third and bottom rows, respectively. Scale bars: $100 \mu \mathrm{m}$, magnified views $50 \mu \mathrm{m}$. 3V, third ventricle. C, Number of OXT-positive cells in the PVN. Control ( $N=3$ mice): $21.18 \pm 4.01$, Caps2 cKO (N=3 mice): $21.92 \pm 3.36$. A total of $8-22$ sections were used for each analysis. Student's $t$ test, $p=0.89$. Data represent the mean \pm SEM. $\boldsymbol{D}$, Ratio of 0 XT- and CAPS2-double-positive cells in the PVN. Control (N $=3$ mice): $59.23 \pm 12.27$, Caps2 cKO ( $N=3$ mice): $1.64 \pm 0.67$. A total of $8-22$ sections were used for each analysis. Student's $t$ test, $p=0.0094$. $* * p<0.01$. Data represent the means \pm SEM.

D; WT: $1347.99 \pm 70.04$ arbitrary units (a.u.); $n=13$; Caps2 KO: $1623.60 \pm 59.44$ a.u.; $n=10$, Student's $t$ test, $p=0.0095]$. The number of mice (Fig. $3 E$; WT: $85.96 \pm 12.01 ; n=13$; Caps $2 \mathrm{KO}$ : $161.79 \pm 20.54 ; n=10$, Student's $t$ test, $p=0.0034)$ and total area (Fig. $3 F$; WT: $508.11 \pm 78.15 \mu \mathrm{m}^{2} ; \quad n=13$; Caps2 KO: $1025.24 \pm 146.09 \mu \mathrm{m}^{2} ; n=10$, Student's $t$ test, $\left.p=0.0038\right)$, but not size (Fig. 3G; WT: $5.61 \pm 0.16 ; n=13$; Caps2 KO: $5.85 \pm 0.13 ; n=10$, Student's $t$ test, $p=0.28$ ), of OXT puncta were increased in Caps $2 \mathrm{KO}$ compared with WT mice. Collectively, these findings indicate that the loss of CAPS2 may cause decreased OXT levels in the blood albeit increased OXT levels in the pituitary and also in the PVN, which further supports our hypothesis regarding a CAPS2-mediated OXT release mechanism.
OXT neuron-specific Caps 2 conditional KO mice also exhibit increased levels of pituitary OXT

To further evaluate the possibility of CAPS2 facilitating OXT release as a regulator of DCV exocytosis, we generated OXT neuron-specific Caps 2 cKO) mice. First, we generated a knock-in (KI) mouse line (termed Oxt-iCre mice) in which the Cre recombinase was integrated into the downstream end of neurophysin1, a C-terminal peptide of the OXT precursor polypeptide, with the insertion of a $2 \mathrm{~A}$ peptide spacer to facilitate endogenous $\mathrm{Oxt}$ promoter-driven expression (Fig. 4A). Cre immunoreactivity was localized to OXT-positive cells in the PVN of Oxt-iCre mice (Fig. $4 B, C$ ). To probe the loxP site-specific Cre recombinase activity, we injected a recombinant adeno-associated virus 5 (AAV5) vector carrying a floxed mCherry, a red fluorescent 
A

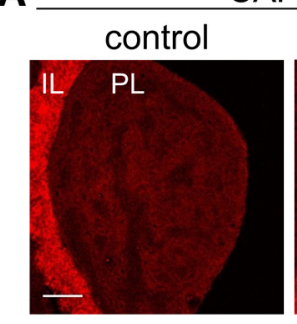

C

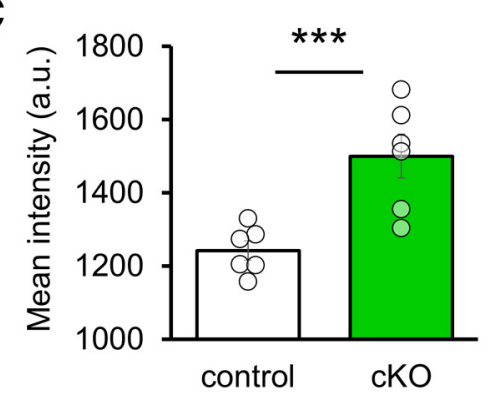

Figure 7. Changes of OXT levels in the pituitary of Caps2 cKO mice. $\boldsymbol{A}$, Representative images of pituitary sections immu-

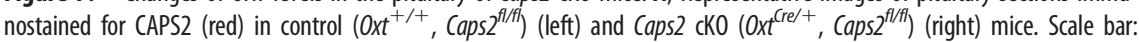
$100 \mu \mathrm{m}$. B, Representative images of pituitary sections immunostained for OXT (green) in control (left) and Caps2 cKO (right) mice. Magnified views of boxed areas $a$ and $b$ on the top row are shown on the bottom row. Scale bars: $100 \mu$ m, top row; $20 \mu \mathrm{m}$, bottom row. $C$, Mean intensity of immunofluorescence intensity for posterior pituitary OXT. Control $(N=13$ mice): $1242.23 \pm 26.30$ a.u., Caps2 cKO ( $N=6$ mice): $1499.79 \pm 59.60$ a.u. Student's $t$ test, $p=0.0027$. A total of $6-9$ pituitary sections were analyzed for each mouse tested. Data represent the means \pm SEM. $* * * p<0.001$. IL, intermediate lobe; PL, posterior lobe.

protein derivative, into the PVN of heterozygous Oxt-iCre mice $\left(O x t^{C r e /+}\right.$; Fig. 5A) and analyzed mCherry expression. Consequently, we could detect mCherry immunoreactivity in the neuroendocrine pathway of the neurohypophysis, i.e., from the PVN, medial eminence, hypothalamic-posterior pituitary stalk, and posterior pituitary lobe (Fig. 5B), as expected. The mCherry immunoreactivity was also observed in several brain regions, including the lateral septum, nucleus accumbens, and ventral tegmental area (Fig. $5 C$ ), which were previously reported as the areas receiving projections from OXT neurons (Knobloch et al., 2012; Grinevich et al., 2016; Hung et al., 2017; Lin et al., 2018; Menon et al., 2018; Hasan et al., 2019) or expressing OXT receptors (Yoshida et al., 2009; Sharma et al., 2019). In the lateral septum and ventral tegmental area, there were CAPS2-positive punctate signals and soma within the region projected by mCherry-positive fibers (Fig. $5 D$ ).

We next generated a Caps 2 floxed mouse line in which exon6 of the Caps2 gene was flanked by the loxP sequence (Fig. 6A). Then, OXT neuron-specific Caps $2 \mathrm{cKO}$ mice were generated by crossing Oxt-iCre heterozygotes $\left(\mathrm{Oxt} \mathrm{Cre}^{\mathrm{C}}\right)$ and Caps2-flox homozygotes $\left(C a p s 2^{f l f l}\right)$. The resultant Cre-carrying Caps2floxed mice $\left(\mathrm{Oxt} \mathrm{Crel+}^{\mathrm{C}}\right.$, Caps $2^{\mathrm{fl} / f l}$ ) showed the absence of CAPS2 immunoreactivity in OXT-positive neurons specifically in the PVN (Fig. 6B) without changes in the number of OXT cells (Fig. 6C; control: $21.18 \pm 4.01$ cells; $n=3$; Caps 2 cKO: $21.92 \pm 3.36$ cells; $n=3$, Student's $t$ test, $p=0.89$ ), exhibiting almost no detectable levels of CAPS2 expression (Fig. $6 D$; control: 59.23 ; 12.27\%; $n=3$; Caps 2 cKO: $1.64 \pm 0.67 \% ; n=3$, Student's $t$ test, $p=0.0094$ ), as expected. Thus, the Caps $2 \mathrm{cKO}$ mouse line was established.

We next analyzed the immunoreactivity for CAPS2 and OXT in the pituitary of Caps $2 \mathrm{cKO}$ mice. CAPS2 immunoreactivity of the Caps2 cKO pituitary was significantly reduced in the posterior lobe but showed similar levels to those of the control pituitary (Fig. 7A), indicating the OXT neuron-specific suppression of CAPS2 expression in cKO mice. Notably, pituitary OXT immunoreactivity was significantly increased in Caps $2 \mathrm{cKO}$ compared with control mice (Fig. 7B,C; control: $1242.23 \pm 26.30$ a.u.; $n=13$; Caps 2 cKO: $1499.79 \pm 59.60$ a.u.; $n=6$, Student's $t$ test, $p=0.0027$ ), again suggesting the accumulation of OXT vesicles in the posterior lobe.

\section{Caps 2 cKO mice exhibit impaired} sociability, which can be ameliorated by administration of exogenous OXT

Finally, we attempted to address the question of whether CAPS2 deficiency influences OXT-associated social behavior. Our previous studies already demonstrated the impairments of social interaction and behavior in Caps2 KO mice (Sadakata et al., 2007b) and mice with the deletion of Caps2 exon 3 (Caps2-deletion of exon3: dex3; Sadakata et al., 2012), although these two model mice were expected to have problems in DCV exocytosis for various peptidergic and catecholaminergic transmitters and peptide hormones in addition to OXT and thus are probably defective in many cellular and behavioral functions. To overcome this issue, we used cKO mice. Except for decreased time spent in the center, which is generally evaluated as a sign of anxiety-like behavior, in terms of total distance and average speed, cKO mice exhibited normal motor ability in an open field, similar to that of control mice (Fig. $8 A-D$ ). In the resident intruder social interaction test (Fig. $8 E-K$ ), Caps 2 cKO mice showed a significant decrease of social interaction duration (approaching, following, or sniffing) with a novel intruder mouse compared

with that of control $\left(\mathrm{Oxt} \mathrm{t}^{+/+}\right.$, Caps $\left.2^{f l f l}\right)$ mice (Fig. 8F; Caps2 cKO: $35.90 \pm 5.42 \mathrm{~s} ; n=7$; control: $76.48 \pm 9.44 \mathrm{~s} ; n=10$, Student's $t$ test, $p=0.011)$. We also confirmed reduced social interaction behavior in Caps2 cKO mice when Oxt-iCre $\left(\mathrm{Oxt} \mathrm{Cre} /+^{+}\right.$, Caps $\left.2^{+/+}\right)$mice were used as a control [Fig. $8 \mathrm{~K}$; OxtiCre heterozygous mice $\left(\mathrm{Oxt} \mathrm{Cre}^{\mathrm{C}+}, \mathrm{Caps}^{+/+}\right): 59.33 \pm 4.64 \mathrm{~s}$; $n=5$; Caps2 cKO mice $\left(O x t^{C r e /+}\right.$, Caps $\left.2^{f l f l}\right): 35.90 \pm 5.42 \mathrm{~s} ; n=7$, Student's $t$ test, $p=0.019]$. In comparison, in the consecutive social interaction test with the same intruder mouse, control mice showed significantly decreased social interaction duration on repeated confrontation with the same intruder, with elevated social interaction when presented with a novel, unfamiliar mouse (Fig. 8G). However, Caps2 cKO mice did not show significant changes in social interaction with either familiar or novel mice (Fig. 8G), although they showed comparable performance to that of control mice in two of three different parameters: latency to first interaction (Fig. $8 H$; Caps 2 cKO: $21.58 \pm 3.38 \mathrm{~s}$; $n=7$; control: $26.57 \pm 3.26 \mathrm{~s} ; n=10$, Student's $t$ test, $p=0.64$ ) and sniffing approach to heads (Fig. 8 I; Caps 2 cKO: $13.92 \pm 2.44 \mathrm{~s} ; n=7$; control: $15.32 \pm 3.18 \mathrm{~s} ; n=10$, Student's $t$ test, $p=0.32$ ) but not tails (Fig. 8J; Caps2 cKO: $19.16 \pm 5.06 \mathrm{~s} ; n=7$; control: $53.69 \pm 8.08 \mathrm{~s}$; $n=10$, Student's $t$ test, $p=0.0053$ ). The results suggest that Caps 2 cKO mice exhibit an impairment in social behavior. 
Because a prior report demonstrated that intranasal administration of exogenous OXT resulted in increased OXT levels in the blood and several brain regions (Neumann et al., 2013), we administered exogenous OXT solution or control saline intranasally to Caps $2 \mathrm{cKO}$ mice before the social interaction test. The OXT-treated group showed much higher social interaction duration than did the saline-treated group (Fig. 9A; Caps2 cKO+OXT: $60.89 \pm 9.16$ s; $n=6$; Caps 2 cKO+saline: $33.95 \pm 6.43 \mathrm{~s} ; n=6$, Student's $t$ test, $p=0.037$ ). Among the three parameters, latency to first interaction (Fig. 9B; Caps2 cKO+OXT: $5.37 \pm 0.83$ s; $n=6$; Caps2 cKO+saline: $6.87 \pm 1.58$ s; $n=6$, Student's $t$ test, $p=0.42$ ) and sniffing approach to heads (Fig. 9C; Caps2 cKO+OXT: $34.63 \pm 7.68$ s; $n=6$; Caps2 cKO+saline: $20.55 \pm 4.54$ s; $n=6$, Student's $t$ test, $p=0.15$ ) did not differ between the two groups, whereas sniffing to tails was significantly increased in Caps2 cKO mice compared with control mice (Fig. 9D; Caps2 cKO+OXT: $30.93 \pm 5.02$ s; $n=6$; Caps 2 cKO+saline: $15.74 \pm 3.29 \mathrm{~s} ; n=6$, Student's $t$ test, $p=0.030)$.

\section{Discussion}

OXT is a neuropeptide/peptide hormone that is considered effective in promoting social behavior in mice and humans (Ross and Young, 2009; Stoop, 2012; Baribeau and Anagnostou, 2015; Johnson and Young, 2017). To elucidate the mechanisms by which OXT release is regulated, in the present study, we focused on the role of CAPS2, a regulator of DCV exocytosis. We showed that CAPS2 is expressed in OXT neurons of the PVN and is substantially localized to the sites (the pituitary for peripheral OXT and several brain areas for central OXT) to which the axons of OXT neurons project and/or OXT is released. We also revealed that Caps2 KO mice had decreased plasma OXT levels albeit increased pituitary OXT levels. In the course of this study, we also generated two mouse lines, Oxt-iCre and Caps2-flox, through which we generated OXT neuron-specific Caps $2 \mathrm{cKO}$ mice. Finally, we elucidated that the removal of CAPS2 specifically from OXT neurons in mice causes impaired social behavior, which could be ameliorated by intranasally administrated exogenous OXT. Together, these results suggested that CAPS2 could regulate the release of OXT as a social neuropeptide/hormone and, thus, is
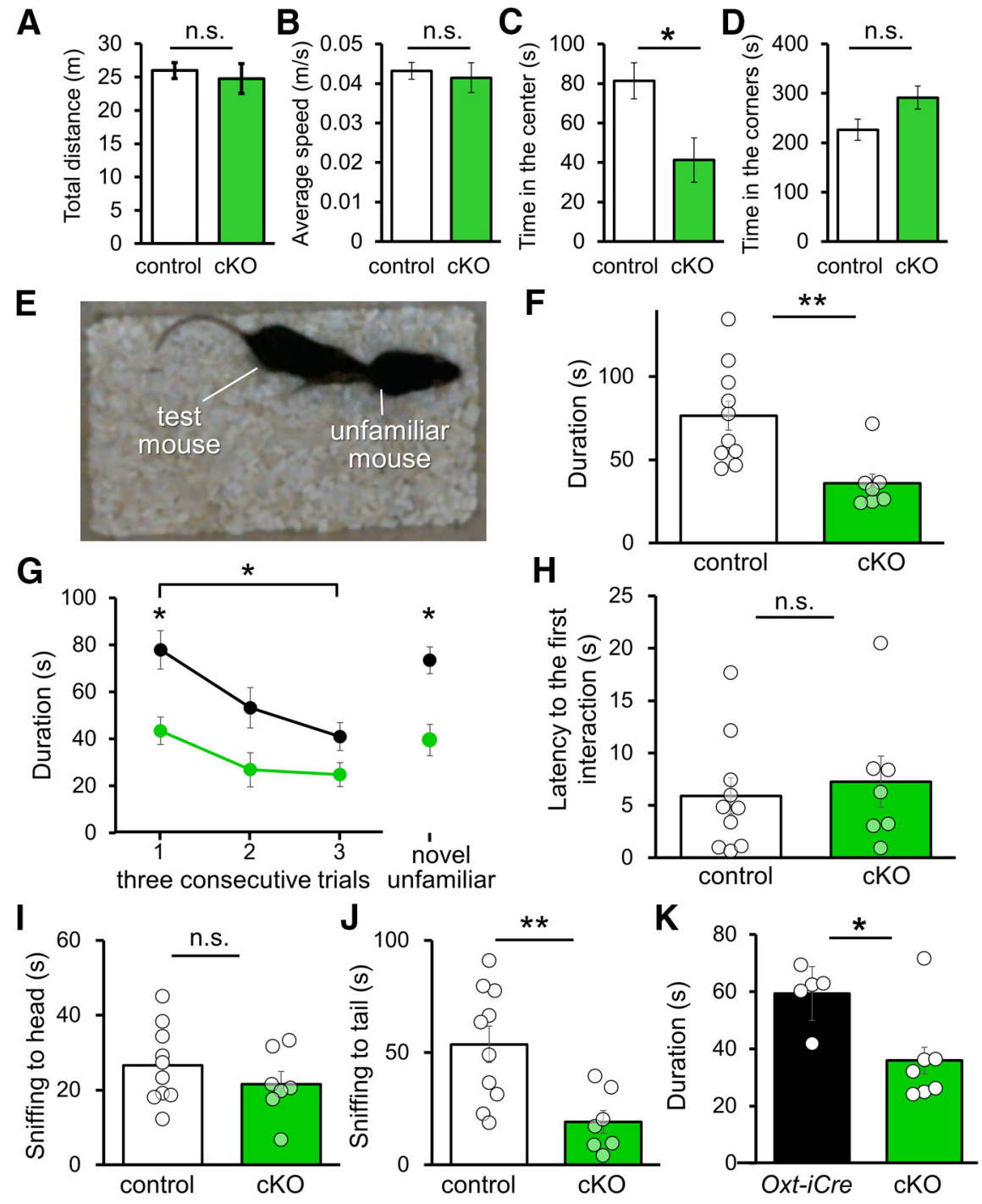

Figure 8. Analysis of social behavior in Caps2 cK0 mice. $A$, Total distance $(s)$ traveled in 10 min open field test. Caps2 cK0 $(N=9$ mice; $24.78 \pm 2.25 \mathrm{~m})$, control $(N=6 ; 25.98 \pm 1.18 \mathrm{~m})$ mice. $p=0.66$ (Student's $t$ test; open circles indicate a data point from an individual mouse). $\boldsymbol{B}$, Average moving speed $(\mathrm{m} / \mathrm{s})$ of mice in $10 \mathrm{~min}$ open field test. Caps 2 cKO $(N=9$ mice; $0.041 \pm 0.0038 \mathrm{~m} / \mathrm{s})$, control $(N=6 ; 0.043 \pm 0.0021 \mathrm{~m} / \mathrm{s}$ ) mice. $p=070$ (Student's $t$ test; open circles indicate a data point from an individual mouse). C, Duration (s) spent at the center of the chamber in a $10 \mathrm{~min}$ open field test. Caps2 cKO ( $N=9$ mice; $41.24 \pm 11.18 \mathrm{~s})$, control $(N=6 ; 81.30 \pm 9.11 \mathrm{~s})$ mice. $p=0.015$ (Student's $t$ test; open circles indicate a data point from an individual mouse). $D$, Duration (s) spent at the corners of the chamber in a 10 min open field test. Caps2 cK0 ( $N=9$ mice; $291.49 \pm 23.49 \mathrm{~s})$, control $(N=6 ; 226.36 \pm 21.41 \mathrm{~s})$ mice. $p=0.16$ (Student's $t$ test; open circles indicate a data point from an individual mouse). $\boldsymbol{E}$, Typical behavior in the reciprocal social interaction test. $\boldsymbol{F}$, Duration (s) of social interaction (including sniffing, approaching, and following) in the three repeated 3 min consecutive trials with 10 min intertrial interval. Caps2 cKO ( $N=7$ mice; $35.90 \pm 5.42 \mathrm{~s})$, control $(N=10 ; 76.48 \pm 9.44 \mathrm{~s})$ mice. $p=0.011$ (Student's $t$ test; open circles indicate a data point from an individual mouse). $\mathbf{G}$, Sniffing duration of the repeated social interaction test. Caps2 cK0 $(N=7)$, control $(N=10)$ mice. Data were analyzed by two-way ANOVA and the Tukey-Kramer post hoc test using StatView and $\mathrm{R} ; p=0.0012$ (repeated two-way ANOVA between control and $\mathrm{KO}$ groups); $p=0.010$ (Tukey-Kramer test between trial 1 and trial 3 of control mice); $p=0.98$ (Tukey-Kramer test between trial 1 and trial 3 of cK0 mice); $p=0.025$ (TukeyKramer test between trial 3 and trial 4 with novel mouse of control mice); $p=0.96$ (Tukey-Kramer test between trial 3 and trial 4 with novel mouse of $\mathrm{CKO}$ mice. $\boldsymbol{H}$, Latency (s) to the first interaction of the first trial of the 3 min social interaction test. Caps2 cKO ( $N=7$ mice; $21.58 \pm 3.38 \mathrm{~s})$, control $(N=10 ; 26.57 \pm 3.26 \mathrm{~s})$ mice. $p=0.64$ (Student's $t$ test; open circles indicate a data point from an individual mouse). $I$, Duration (s) of interaction with the head in the first 3 min trial. Caps 2 CKO ( $N=7$ mice; $13.92 \pm 2.44 \mathrm{~s}$ ), control $(N=10 ; 15.32 \pm 3.18 \mathrm{~s}$ ) mice. $p=0.32$ (Student's $t$ test; open circles indicate a data point from an individual mouse). $J$, Duration (s) of anogenital interaction in the first 3 min trial. Caps2 cKO ( $N=7$ mice; $19.16 \pm 5.06 \mathrm{~s})$, control $(N=10 ; 53.69 \pm 8.08 \mathrm{~s}$ ) mice. $p=0.0053$ (Student's $t$ test; open circles indicate a data point from an individual mouse). $\boldsymbol{K}$, Duration (s) of reciprocal interaction (including sniffing, approaching, and following) in the 3 min trial was compared between Oxt-iCre heterozygous mice $\left(0 x t^{(\text {re } /+}\right.$, Caps $^{+/+} ; N=5$ mice; $59.33 \pm 4.64$ s) and Caps2 cKO mice $\left(0 x t^{\left(r^{r e} /+\right.}\right.$, Caps $\left.2^{f / f f} ; N=7 ; 35.90 \pm 5.42 \mathrm{~s}\right) . p=0.019$ indicates significant difference between two groups (Student's $t$ test; open circles indicate a data point from an individual mouse). Data represent the means $\pm S E M, * p<0.05$; $* * p<0.01$, n.s., Not significant. 
A

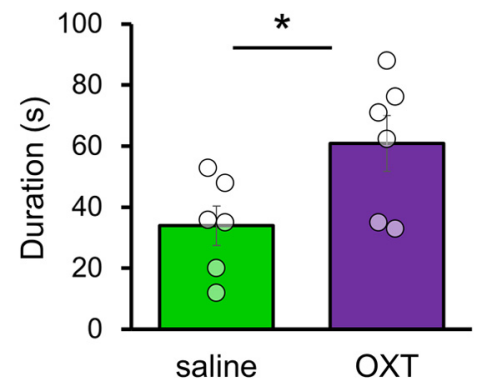

C

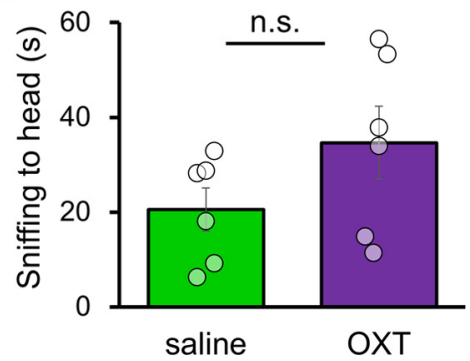

B

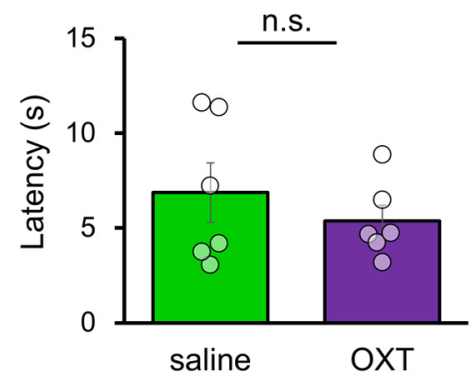

D

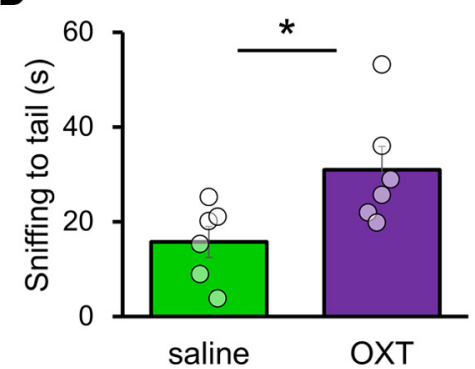

Figure 9. Effect of intranasal administration of exogenous OXT on the social behavior of Caps2 cK0 mice. A, Sniffing duration in the $3 \mathrm{~min}$ reciprocal social interaction test at $20 \mathrm{~min}$ after the intranasal OXT or saline administration. Caps2 cK0 + OXT ( $n=6$ mice; $60.89 \pm 9.16$ s), Caps2 cK0 + saline ( $n=6$ mice; $33.95 \pm 6.43 \mathrm{~s}$ ). $* p=0.037$ indicates significant difference between two groups (Student's $t$ test; open circles indicate a data point from an individual mouse). $\boldsymbol{B}$, Latency $(s)$ to the first interaction of the trial of the $3 \mathrm{~min}$ social interaction test. Caps 2 cKO $+0 X T$ ( $n=6$ mice; $5.37 \pm 0.83 \mathrm{~s}$ ), Caps 2 cKO + saline ( $n=6$ mice; $6.87 \pm 1.58 \mathrm{~s}$ ). $p=0.42$ indicates significant difference between two groups (Student's $t$ test; open circles indicate a data point from an individual mouse). $C$, Duration (s) of interaction to the body in the 3 min trial. CAPS2 cKO+0XT ( $n=6$ mice; $34.63 \pm 7.68 \mathrm{~s})$, Caps2 cK0 + saline ( $n=6$ mice; $20.55 \pm 4.54$ s). $p=0.15$ indicates no significant difference between two groups (Student's $t$ test; open circles indicate a data point from an individual mouse). D, Duration (s) of anogenital interaction in the 3 min trial. Caps2 cK0 + 0XT ( $n=6$ mice; $30.93 \pm 5.02$ s), Caps2 cK0 + saline ( $n=6$ mice; $15.74 \pm 3.29$ s). $p=0.030$ indicates significant difference between two groups (Student's $t$ test; open circles indicate a data point from an individual mouse). Student $t$ test and two-way ANOVA were used to compare the experimental groups. The Tukey-Kramer test was used for post hoc analysis. Data represent the means \pm SEM. $* p<0.05$. n.S., Not significant.

associated with the cellular basis required for the expression of social behavior.

In the present study, we report the first evidence that CAPS2 facilitates DCV exocytosis of OXT, a sociality-associated secretory substance, thereby potentially explaining the neural and social behavior deficits associated with CAPS2 deficiency in mice (Sadakata et al., 2007b, 2012) and identification of Caps2 molecular variation in ASD (Sadakata et al., 2007b; Christian et al., 2008; Marshall et al., 2008; Stein et al., 2010; Okamoto et al., 2011; Girirajan et al., 2013; Bonora et al., 2014; Aristidou et al., 2017; Grabowski et al., 2017; Grove et al., 2019). Our study also showed that CAPS2 is expressed in PVN cells expressing AVP. As AVP is also known to be associated with social behavior control (Stoop, 2012; Baribeau and Anagnostou, 2015; Johnson and Young, 2017), the possible connection of CAPS2-mediated AVP release with social behavior warrants further investigation.

CAPS2 was localized to OXT-producing cells in the hypothalamic PVN, from which the pituitary and several brain areas such as the lateral septum and ventral tegmental area (VTA) receive OXT nerve projections. From the posterior pituitary, peripheral OXT is released as a neurohypophyseal hormone into the blood circulation. In the other brain regions to which PVN OXT neurons project, central OXT is likely released to activate the central OXT signaling pathway (Knobloch et al., 2012; Marlin et al., 2015; Hung et al., 2017; Lin et al., 2018; Hasan et

al., 2019). We previously demonstrated that Caps 2 mRNA and protein are expressed in many brain regions, including the septal regions, striatum, midbrain VTA, and substantia nigra pars compacta in mice (Sadakata et al., 2006), some of which have been implicated in social behavior. In the present study, our data supported the involvement of CAPS2 in peripheral OXT release; however, we were unable to provide direct evidence of its role in central OXT release. Thus, although our findings support the role of peripheral OXT signaling in social behavior in addition to that of central OXT, the possibility of CAPS2-mediated central OXT release remains to be verified. The quantification of OXT contents by ELISA showed that the blood plasma levels were significantly lower in Caps2 KO than in WT mice. In contrast, OXT immunoreactivity in the posterior pituitary was higher in both Caps $2 \mathrm{KO}$ and $\mathrm{cKO}$ mice than in their respective control mice. Together, these observations suggest that CAPS2 promotes OXT secretion from the pituitary to the blood, although it remains necessary to obtain direct evidence regarding CAPS2-mediated OXT release, such as imaging analysis. The accumulation levels of hypothalamic PVN and pituitary OXT were considerably higher in both Caps2 $\mathrm{KO}$ and cKO mice than those in their respective controls, whereas reduced levels of plasma OXT in Caps $2 \mathrm{KO}$ mice were significantly but slightly lower, at $75.8 \%$ of that of WT mice. These results raise a question regarding how levels of blood OXT are functionally associated with social behavior. Notably, it has been reported that brain OXT levels were increased after nasal as well as intraperitoneal administration of OXT in rats and mice (Neumann et al., 2013) and that the release of central OXT was triggered by social stimuli, including sexual, aggressive, and stressful events (Veenema and Neumann, 2008), suggesting activity-dependent changes of blood OXT and brain OXT levels, both of which appear to be linked (Crockford et al., 2014). As CAPS family proteins play a facilitating role at the priming step of activity-dependent DCV exocytosis (Grishanin et al., 2002; Speidel et al., 2005; Liu et al., 2008; Shinoda et al., 2011; Kabachinski et al., 2016), we suggest that social contexts activate CAPS2-mediated DCV exocytosis, consequently inducing an increase in OXT levels beyond their basal levels in the blood and/or brain. Nevertheless, the present study did not provide direct evidence regarding CAPS2-dependent central OXT release from the axon terminals of hypothalamic PVN neurons to brain regions, although OXT levels were enhanced in the hypothalamic PVN of Caps2 KO mice. In addition, there is a possible role of CAPS2 in autocrine/paracrine regulation of OXT neurons and their neighboring cells by somatodendritic OXT release (Brown et al., 2020). Further study will therefore be needed to elucidate this issue.

The previous studies on Oxt $\mathrm{KO}$ mice completely lacking OXT release suggest that OXT is necessary for specific sociability, including social memory, investigation, and aggressiveness in mice (Ferguson et al., 2000; Crawley et al., 2007; Lazzari et al., 2013). In the resident intruder tests, Caps 2 cKO mice showed 
lower social interaction duration compared with the control mice. In the consecutive social interaction test with the same intruder mouse, Caps $2 \mathrm{cKO}$ mice showed similar levels of interaction behavior with the intruder mouse despite repeatedly confronting the same mouse. When presented with a novel, unfamiliar mouse, Caps 2 cKO mice did not show significant changes in social interaction with either familiar or novel mice. Oxt KO mice also exhibited no decline of social interaction during repeated pairing with the same familiar mice or during the presentation of a novel unfamiliar mice (Ferguson et al., 2000). Together, our results suggest that Caps2 cKO mice have similar behavioral phenotypes as Oxt $\mathrm{KO}$ mice that were previously suggested to have impairments specific to social memory (Ferguson et al., 2000; Crawley et al., 2007). Finally, our suggestion that CAPS2 regulates the release of social peptide OXT is strongly supported by the evidence that intranasal treatment with exogenous OXT demonstrated ameliorative effects on the decreased social behavior of Caps $2 \mathrm{cKO}$ mice.

In conclusion, we demonstrated that CAPS2 is expressed in the neurohypophyseal OXT pathway from the PVN OXT neurons to their axonal projection site, the posterior pituitary, and is also present in PVN OXT neurons that project to several brain regions. The deficiency of CAPS2 in mice causes decreased plasma OXT levels and, conversely, elevated pituitary OXT levels, suggesting the functional role of CAPS2 in regulating proper OXT release. In this study, we succeeded in generating a mouse line harboring OXT neuron-specific deletion of CAPS2 (Caps2 cKO mice). Caps2 cKO mice display impaired social behavior similar to that of Oxt KO mice, and their impaired social behavior could be ameliorated by treatment with exogenous OXT. Collectively, our findings suggest that CAPS2 is a critical factor for OXT release, consequently being associated with social behavior. Further evaluation of the CAPS2-mediated OXT release mechanism will help identify whether this process may serve as a potential target for ASD therapy.

\section{References}

Aristidou C, Koufaris C, Theodosiou A, Bak M, Mehrjouy MM, Behjati F, Tanteles G, Christophidou-Anastasiadou V, Tommerup N, Sismani C (2017) Accurate breakpoint mapping in apparently balanced translocation families with discordant phenotypes using whole genome mate-pair sequencing. PLoS One 12:e0169935.

Auyeung B, Lombardo MV, Heinrichs M, Chakrabarti B, Sule A, Deakin JB, Bethlehem RA, Dickens L, Mooney N, Sipple JA, Thiemann P, BaronCohen S (2015) Oxytocin increases eye contact during a real-time, naturalistic social interaction in males with and without autism. Transl Psychiatry 5:e507.

Baribeau DA, Anagnostou E (2015) Oxytocin and vasopressin: linking pituitary neuropeptides and their receptors to social neurocircuits. Front Neurosci 9:335.

Bonora E, Graziano C, Minopoli F, Bacchelli E, Magini P, Diquigiovanni C, Lomartire S, Bianco F, Vargiolu M, Parchi P, Marasco E, Mantovani V, Rampoldi L, Trudu M, Parmeggiani A, Battaglia A, Mazzone L, Tortora G, Maestrini E, Seri M, et al. (2014) Maternally inherited genetic variants of CADPS2 are present in autism spectrum disorders and intellectual disability patients. EMBO Mol Med 6:795-809.

Brown CH, Ludwig M, Tasker JG, Stern JE (2020) Somato-dendritic vasopressin and oxytocin secretion in endocrine and autonomic regulation. J Neuroendocrinol 32:e12856.

Christian SL, Brune CW, Sudi J, Kumar RA, Liu S, Karamohamed S, Badner JA, Matsui S, Conroy J, McQuaid D, Gergel J, Hatchwell E, Gilliam TC, Gershon ES, Nowak NJ, Dobyns WB, Cook EH Jr (2008) Novel submicroscopic chromosomal abnormalities detected in autism spectrum disorder. Biol Psychiatry 63:1111-1117.

Crawley JN, Chen T, Puri A, Washburn R, Sullivan TL, Hill JM, Young NB, Nadler JJ, Moy SS, Young LJ, Caldwell HK, Young WS (2007) Social approach behaviors in oxytocin knockout mice: comparison of two independent lines tested in different laboratory environments. Neuropeptides 41:145-163.

Crockford C, Deschner T, Ziegler TE, Wittig RM (2014) Endogenous peripheral oxytocin measures can give insight into the dynamics of social relationships: a review. Front Behav Neurosci 8:68.

Ferguson JN, Young LJ, Hearn EF, Matzuk MM, Insel TR, Winslow JT (2000) Social amnesia in mice lacking the oxytocin gene. Nat Genet 25:284-288.

Girirajan S, Dennis MY, Baker C, Malig M, Coe BP, Campbell CD, Mark K, Vu TH, Alkan C, Cheng Z, Biesecker LG, Bernier R, Eichler EE (2013) Refinement and discovery of new hotspots of copy-number variation associated with autism spectrum disorder. Am J Hum Genet 92:221-237.

Grabowski PAP, Bello AF, Rodrigues DL, Forbeci MJ, Motter V, Raskin S (2017) Deletion involving the 7q31-32 band at the CADPS2 gene locus in a patient with autism spectrum disorder and recurrent psychotic syndrome triggered by stress. Case Rep Psychiatry 2017:4254152.

Grinevich V, Knobloch-Bollmann HS, Eliava M, Busnelli M, Chini B (2016) Assembling the puzzle: pathways of oxytocin signaling in the brain. Biol Psychiatry 79:155-164.

Grishanin RN, Klenchin VA, Loyet KM, Kowalchyk JA, Ann K, Martin TF (2002) Membrane association domains in Ca2+-dependent activator protein for secretion mediate plasma membrane and dense-core vesicle binding required for $\mathrm{Ca} 2+$-dependent exocytosis. J Biol Chem 277:22025-22034.

Grove J, Ripke S, Als TD, Mattheisen M, Walters RK, Won H, Pallesen J, Agerbo E, Andreassen OA, Anney R, Awashti S, Belliveau R, Bettella F, Buxbaum JD, Bybjerg-Grauholm J, Bækvad-Hansen M, Cerrato F, Chambert K, Christensen JH, Churchhouse C, Dellenvall K, Demontis D, De Rubeis S, Devlin B, Djurovic S, Dumont AL, Goldstein JI, Hansen CS, Hauberg ME, et al. (2019) Identification of common genetic risk variants for autism spectrum disorder. Nat Genet 51:431-444.

Hasan MT, Althammer F, Silva da Gouveia M, Goyon S, Eliava M, Lefevre A, Kerspern D, Schimmer J, Raftogianni A, Wahis J, Knobloch-Bollmann HS, Tang Y, Liu X, Jain A, Chavant V, Goumon Y, Weislogel J-M, Hurlemann R, Herpertz SC, Pitzer C, et al. (2019) A fear memory engram and its plasticity in the hypothalamic oxytocin system. Neuron 103:133146.e8.

Hung LW, Neuner S, Polepalli JS, Beier KT, Wright M, Walsh JJ, Lewis EM, Luo L, Deisseroth K, Dölen G, Malenka RC (2017) Gating of social reward by oxytocin in the ventral tegmental area. Science 357:1406-1411.

Johnson ZV, Young LJ (2017) Oxytocin and vasopressin neural networks: implications for social behavioral diversity and translational neuroscience. Neurosci Biobehav Rev 76:87-98.

Kabachinski G, Kielar-Grevstad DM, Zhang X, James DJ, Martin TF (2016) Resident CAPS on dense-core vesicles docks and primes vesicles for fusion. Mol Biol Cell 27:654-668.

Knobloch HS, Charlet A, Hoffmann LC, Eliava M, Khrulev S, Cetin AH, Osten P, Schwarz MK, Seeburg PH, Stoop R, Grinevich V (2012) Evoked axonal oxytocin release in the central amygdala attenuates fear response. Neuron 73:553-566.

Kosfeld M, Heinrichs M, Zak PJ, Fischbacher U, Fehr E (2005) Oxytocin increases trust in humans. Nature 435:673-676.

Lazzari VM, Becker RO, de Azevedo MS, Morris M, Rigatto K, Almeida S, Lucion AB, Giovenardi M (2013) Oxytocin modulates social interaction but is not essential for sexual behavior in male mice. Behav Brain Res 244:130-136.

Lin YT, Hsieh TY, Tsai TC, Chen CC, Huang CC, Hsu KS (2018) Conditional deletion of hippocampal CA2/CA3a oxytocin receptors impairs the persistence of long-term social recognition memory in mice. J Neurosci 38:1218-1231.

Liu Y, Schirra C, Stevens DR, Matti U, Speidel D, Hof D, Bruns D, Brose N, Rettig J (2008) CAPS facilitates filling of the rapidly releasable pool of large dense-core vesicles. J Neurosci 28:5594-5601.

Marlin BJ, Mitre M, D’Amour JA, Chao MV, Froemke RC (2015) Oxytocin enables maternal behaviour by balancing cortical inhibition. Nature 520:499-504.

Marshall CR, Noor A, Vincent JB, Lionel AC, Feuk L, Skaug J, Shago M, Moessner R, Pinto D, Ren Y, Thiruvahindrapduram B, Fiebig A, Schreiber S, Friedman J, Ketelaars CEJ, Vos YJ, Ficicioglu C, Kirkpatrick S, Nicolson R, Sloman L, et al. (2008) Structural variation of chromosomes in autism spectrum disorder. Am J Hum Genet 82:477-488. 
Menon R, Grund T, Zoicas I, Althammer F, Fiedler D, Biermeier V, Bosch OJ, Hiraoka Y, Nishimori K, Eliava M, Grinevich V, Neumann ID (2018) Oxytocin signaling in the lateral septum prevents social fear during lactation. Curr Biol 28:1066-1078.e6.

Neumann ID, Maloumby R, Beiderbeck DI, Lukas M, Landgraf R (2013) Increased brain and plasma oxytocin after nasal and peripheral administration in rats and mice. Psychoneuroendocrinology 38:1985-1993.

Okamoto N, Hatsukawa Y, Shimojima K, Yamamoto T (2011) Submicroscopic deletion in 7q31 encompassing CADPS2 and TSPAN12 in a child with autism spectrum disorder and PHPV. Am J Med Genet A 155a:1568-1573.

Peñagarikano O, Lázaro MT, Lu XH, Gordon A, Dong H, Lam HA, Peles E, Maidment NT, Murphy NP, Yang XW, Golshani P, Geschwind DH (2015) Exogenous and evoked oxytocin restores social behavior in the Cntnap2 mouse model of autism. Sci Transl Med 7:271-ra278.

Ross HE, Young LJ (2009) Oxytocin and the neural mechanisms regulating social cognition and affiliative behavior. Front Neuroendocrinol 30:534547.

Sadakata T, Mizoguchi A, Sato Y, Katoh-Semba R, Fukuda M, Mikoshiba K, Furuichi T (2004) The secretory granule-associated protein CAPS2 regulates neurotrophin release and cell survival. J Neurosci 24:43-52.

Sadakata T, Itakura M, Kozaki S, Sekine Y, Takahashi M, Furuichi T (2006) Differential distributions of the $\mathrm{Ca} 2+$-dependent activator protein for secretion family proteins (CAPS2 and CAPS1) in the mouse brain. J Comp Neurol 495:735-753.

Sadakata T, Kakegawa W, Mizoguchi A, Washida M, Katoh-Semba R, Shutoh F, Okamoto T, Nakashima H, Kimura K, Tanaka M, Sekine Y, Itohara S, Yuzaki M, Nagao S, Furuichi T (2007a) Impaired cerebellar development and function in mice lacking CAPS2, a protein involved in neurotrophin release. J Neurosci 27:2472-2482.

Sadakata T, Washida M, Iwayama Y, Shoji S, Sato Y, Ohkura T, KatohSemba R, Nakajima M, Sekine Y, Tanaka M, Nakamura K, Iwata Y, Tsuchiya KJ, Mori N, Detera-Wadleigh SD, Ichikawa H, Itohara S, Yoshikawa T, Furuichi T (2007b) Autistic-like phenotypes in Cadps2knockout mice and aberrant CADPS2 splicing in autistic patients. J Clin Invest 117:931-943.

Sadakata T, Shinoda Y, Oka M, Sekine Y, Sato Y, Saruta C, Miwa H, Tanaka M, Itohara S, Furuichi T (2012) Reduced axonal localization of a Caps2 splice variant impairs axonal release of BDNF and causes autistic-like behavior in mice. Proc Natl Acad Sci U S A 109:21104-21109.

Sgritta M, Dooling SW, Buffington SA, Momin EN, Francis MB, Britton RA, Costa-Mattioli M (2019) Mechanisms underlying microbial-mediated changes in social behavior in mouse models of autism spectrum disorder. Neuron 101:246-259.e6.

Sharma K, LeBlanc R, Haque M, Nishimori K, Reid MM, Teruyama R (2019) Sexually dimorphic oxytocin receptor-expressing neurons in the preoptic area of the mouse brain. PLoS One 14:e0219784.

Shinoda Y, Sadakata T, Nakao K, Katoh-Semba R, Kinameri E, Furuya A, Yanagawa Y, Hirase H, Furuichi T (2011) Calcium-dependent activator protein for secretion 2 (CAPS2) promotes BDNF secretion and is critical for the development of GABAergic interneuron network. Proc Natl Acad Sci U S A 108:373-378.

Speidel D, Bruederle CE, Enk C, Voets T, Varoqueaux F, Reim K, Becherer U, Fornai F, Ruggieri S, Holighaus Y, Weihe E, Bruns D, Brose N, Rettig J (2005) CAPS1 regulates catecholamine loading of large dense-core vesicles. Neuron 46:75-88.

Stein JL, Hua X, Lee S, Ho AJ, Leow AD, Toga AW, Saykin AJ, Shen L, Foroud T, Pankratz N, Huentelman MJ, Craig DW, Gerber JD, Allen AN, Corneveaux JJ, Dechairo BM, Potkin SG, Weiner MW, Thompson P (2010) Voxelwise genome-wide association study (vGWAS). Neuroimage 53:1160-1174.

Stoop R (2012) Neuromodulation by oxytocin and vasopressin. Neuron 76:142-159.

Takayanagi Y, Yoshida M, Bielsky IF, Ross HE, Kawamata M, Onaka T, Yanagisawa T, Kimura T, Matzuk MM, Young LJ, Nishimori K (2005) Pervasive social deficits, but normal parturition, in oxytocin receptor-deficient mice. Proc Natl Acad Sci U S A 102:16096-16101.

Veenema AH, Neumann ID (2008) Central vasopressin and oxytocin release: regulation of complex social behaviours. Prog Brain Res 170:261-276.

Yoshida M, Takayanagi Y, Inoue $\mathrm{K}$, Kimura $\mathrm{T}$, Young LJ, Onaka T, Nishimori K (2009) Evidence that oxytocin exerts anxiolytic effects via oxytocin receptor expressed in serotonergic neurons in mice. J Neurosci 29:2259-2271.

Zhang HF, Dai YC, Wu J, Jia MX, Zhang JS, Shou XJ, Han SP, Zhang R, Han JS (2016) Plasma Oxytocin and Arginine-Vasopressin Levels in Children with Autism Spectrum Disorder in China: associations with Symptoms. Neurosci Bull 32:423-432. 